\title{
Estimation of the volume of production of turbine vapor of a fuel boiler with stochastic exogenous factors
}

\author{
Seyit Kerimkhulle ${ }^{*}$, Gulmira Azieva ${ }^{1}$, Ainur Saliyeva ${ }^{2}$, and Ayagoz Mukhanova ${ }^{3}$ \\ ${ }^{1}$ L.N. Gumilyov Eurasian National University, 1, Satpaev str., 010000, Nur-Sultan, Kazakhstan \\ ${ }^{2}$ KMG Engineering LLP, 17, Kabanbai batyr str., 010000, Nur-Sultan, Kazakhstan \\ ${ }^{3}$ Al-Farabi Kazakh National University, 71, al-Farabi ave., 050040, Almaty, Kazakhstan
}

\begin{abstract}
The paper is based on the analysis and review of the conceptual principles, methodology and practice of modelling water-steam processes of a fuel boiler of power plants; implementation of algorithms and models of the theory of system dynamics, white and pink noise; using computational technologies of difference-scheme equations and numerical integration methods, the values of the input parameters of the production of turbine vapor of the fuel boiler are determined), which, regardless of stochastic exogenous factors, provide stable states of the necessary and model volumes, norms of water intake and output of turbine vapor of the fuel boiler.
\end{abstract}

\section{Introduction}

An urgent problem for the formation of an intelligent system for evaluating the production of turbine vapor by exogenous stochastic factors in the chain of technological processes of heat power plants is the reliable (stable) provision of tools for managing the state of fuel boiler facilities. In particular, it is possible to solve this problem of steam generation of a fuel boiler by creating a clear system of information environment for managing internal resources and external connections of complex objects [1] of water-steam processes.

We conduct a review of the literature on the following studies, for example, the article [2] considers the problem of balancing supply and demand in the Saxony-Anhalt's energy system and issues of ensuring flexibility in energy systems are studied in four categories: flexible generators, demand management, storage, and transmission of electricity at $\mathrm{X}$, and developed a practical method for quantifying and comparing the technical capabilities of flexibility options at the regional level.

In article [3], the concept of application flexibility to energy systems for operational planning of the transmission network in a market environment is proposed. A specific and unique definition of the flexibility of the transmission system in relation to generation changes is proposed. A mathematical model and a method for calculating some indicators of the flexibility of a volumetric system are also presented.

In [4], an assessment of the industrial water consumption of the electronic industry of the People's Republic of China is given and the geolocation of semiconductor manufacturing enterprises by water intakes at various spatial scales is mapped. On the other hand, geolocation of the data also made it possible to calculate the water intake weighted by the deficit to identify a specific catchment area and map hot spots of industrial water use.

Article [5] presents a thermodynamic simulation of the steam compression cycle based on neural network approach. A generalized radial basis function is used for the network, which generates the predicted current state as the output of the network. The trained network is confirmed by untrained data and shows to a satisfactory extent all the characteristics of the steam compression cycle process for an air-water heat pump.

Article [6] analyzes the consequences of probable hydrological fluctuations during climate change in anticipation of water availability and its variability. Conditions for optimal distribution of water resources are determined to maximize the overall social benefit from water use. The results show that the active participation of the regulator in the water market leads to excellent results in terms of both greater overall social benefits and more water available for environmental purposes in the context of climate change.

Article [7] analyzes for a long-term forecast period all available simulated climate scenarios by checking the scenarios using historical data and assessing the impact on water resources over the next 30 years using a hydrological model proposed by the Spanish Agency of Meteorology (AEMET) for the geographical area covered by the river basin, located in eastern Spain.

Article [8] presents the groundwater stability index, which offers a new combination of characteristics in which the signal-to-noise ratio of long-term trends is used, estimated using reliable regression and the

\footnotetext{
* Corresponding author: kerimkhulle@gmail.com
} 
concentration of the main pollutant causing anxiety of the population.

Article [9] substantiates the methodology for assessing the impact of changes related to groundwater and climate change on the needs for basic and environmental runoff (EFN) and showed that under average meteorological conditions, the basic runoff in the main streams still meets the requirements of EFN. It also determined that anthropogenic changes have a significant impact: drainage caused a $25-40 \%$ reduction in base runoff, groundwater abstraction caused 5-28\%, and climate change will potentially cause an additional $33-70 \%$ reduction by 2050 .

\section{Materials and Methods}

Conceptual principles, methodology and practice of modelling water-steam processes of a fuel boiler of power plants [2-13], algorithms and object-oriented program codes for data processing of problems of system dynamics, white and pink noise $[1,15]$, as well as models of difference-scheme equations [14, also see (1) and BoilerB in Fig. 1] and methods of numerical integration, such as Euler and Runge-Kutta methods.

\section{Results and Discussion}

Let BoilerB $B_{t},-$ an endogenous indicator of the volume of turbine steam production of a fuel boiler for a period of time $t=1,2, \ldots$, then the equilibrium state of the watersteam mass of the fuel boiler depends on the difference of exogenous production indicators between WaterRateWR $R_{t}$, - the norms of water intake and SteamRateSR $\mathrm{t}_{\mathrm{t}}$, - the norms of steam production as a difference-scheme equation of the first order:

$$
\frac{\text { BoilerB }_{\mathrm{t}}}{\mathrm{dt}}=\text { WaterRateWR }_{\mathrm{t}}-\text { SteamRateSR }_{\mathrm{t}}
$$

and the necessary initial volume of turbine steam production:

$$
\text { BoilerB }\left.\right|_{\mathrm{t}=0}=\text { DesiredBoilerB_, }_{\mathrm{t}}
$$

$$
\text { Where: }
$$

- WaterRateWR $\mathrm{R}_{\mathrm{t}}$, - the water intake rate of the input to the water-steam fuel boiler system in the equation of state (1) is defined as the maximum value between "zero" and the required water intake rate:

Water Rate $W R_{t}=\max \left(0\right.$, DesiredWaterRateDWR $\left.\mathrm{R}_{\mathrm{t}}\right)$

- DesiredWaterRateDWR $\mathrm{R}_{\mathrm{t}},-$ the required volume of the water intake rate into the water-steam fuel boiler system in (3) consists of the sum of the expected volume of the steam rate and the adjusted volume of turbine steam production in accordance with the desired level:

$$
\begin{aligned}
& \text { DesiredWaterRateDWR }_{\mathrm{t}} \\
& =\text { ExpectedSteamRateES }_{\mathrm{t}} \\
& \text { +AdjustmentForBoilerAB }
\end{aligned}
$$

- AdjustmentForBoilerAB $\mathrm{B}_{\mathrm{t}}$, - the adjustment of the volume of production of turbine vapor of the fuel boiler in (4) is proportional to the difference between the necessary and the actual volume of turbine steam production:
AdjustmentForBoiler $\mathrm{AB}_{\mathrm{t}}$

$$
=\frac{\text { DesiredBoilerB_-Boiler } B_{t}}{\text { BoilerAdjustmentTimeBAT }} \text {, }
$$

BoilerAdjustmentTimeBAT $=3$,

- ExpectedSteamRateES $\mathrm{S}_{\mathrm{t}},-$ the expected volume of the steam norm is assumed to be equal to the actual level of the developed norm of turbine vapor of a watersteam fuel boiler:

ExpectedSteamRateES $_{\mathrm{t}}=$ SteamRateSR $_{\mathrm{t}}$,

- SteamRateSR $\mathrm{t}_{\mathrm{t}}$, - the norms of turbine vapor are assumed to be equal to the volume of production of turbine vapor of the fuel boiler in (1) with a constant average production life cycle:

$$
\begin{array}{r}
\text { SteamRateSR } \mathrm{t}_{\mathrm{t}}=\frac{\text { BoilerB }_{\mathrm{t}}}{\text { AverageLife }}, \\
\text { AverageLife }=8,
\end{array}
$$

- DesiredBoilerB_, - the exogenous volume of the expected production of turbine vapor of a fuel boiler is determined as the product of the initial volume of the expected steam and the input exogenous variable:

$$
\begin{aligned}
& \text { DesiredBoilerB_ } \\
& ={\text { InitialDesiredBoiler } * \text { Input }_{\mathrm{t}},} \text { InitialDesiredBoiler }=100,
\end{aligned}
$$

- Input $t_{t},-$ a dimensionless variable that provides simulations of input, for example, step, pulse, sinusoidal data and PinkNoise random noise data:

$$
\begin{aligned}
& \text { Input }_{t}=1+\operatorname{step}(\text { StepHeight, StepTime) } \\
& +\left(\frac{\text { PulseQuantity }}{\text { TIME_STEP }}\right) \cdot \operatorname{pulse}\left(\begin{array}{c}
\text { PulseTime, } \\
\text { TIME }_{\text {STEP }}
\end{array}\right) \\
& \operatorname{ramp}\left(\begin{array}{c}
\text { RampSlope, } \\
\text { RampStartTime, } \\
\text { RampEndTime }
\end{array}\right) \\
& + \text { SineAmplitude } \cdot \sin \left(2 \cdot 3.14159 \cdot \frac{\text { time }(~)}{\text { SinePeriod }}\right) \\
& +\operatorname{step}(1, \text { NoiseStartTime) } * \text { PinkNoise , }
\end{aligned}
$$

and the value of constants:

$$
\begin{aligned}
& \text { RampStartTime }=1, \text { RampEndTime }=1 \mathrm{e} \\
& +009, \text { StepTime }=1, \text { SinePeriod }=50, \\
& \text { PulseTime }=1, \text { NoiseStartTime }=1 .
\end{aligned}
$$

The implementation of the above model (1)-(10) hold in the environment of the software product AnyLogic 8.7.6 [15] for this input put the following values:

- PulseQuantity, - number of pulses $=0.38$,

- SineAmplitude, - amplitude sine $=0.14$,

- StepHeight, - step height $=0.5$,

- RampSlope, gradient ramp $=0.00$,

- NoiseStandardDeviation, is the standard deviation of the noise $=0.21$ and will be used the data obtained in the result of this simulation white and pink noise random variables.

The obtained system management solution-finding the values of the input parameters of development vapor turbine fuel boiler (see Fig. 1) that regardless of stochastic exogenous factors provide a reliable (stable) States of the water-steam process:

- required model and the volume of vapor turbine (see Fig. 2), 
- the rules of water flow and the exit vapor turbine fuel boiler (see Fig. 3).

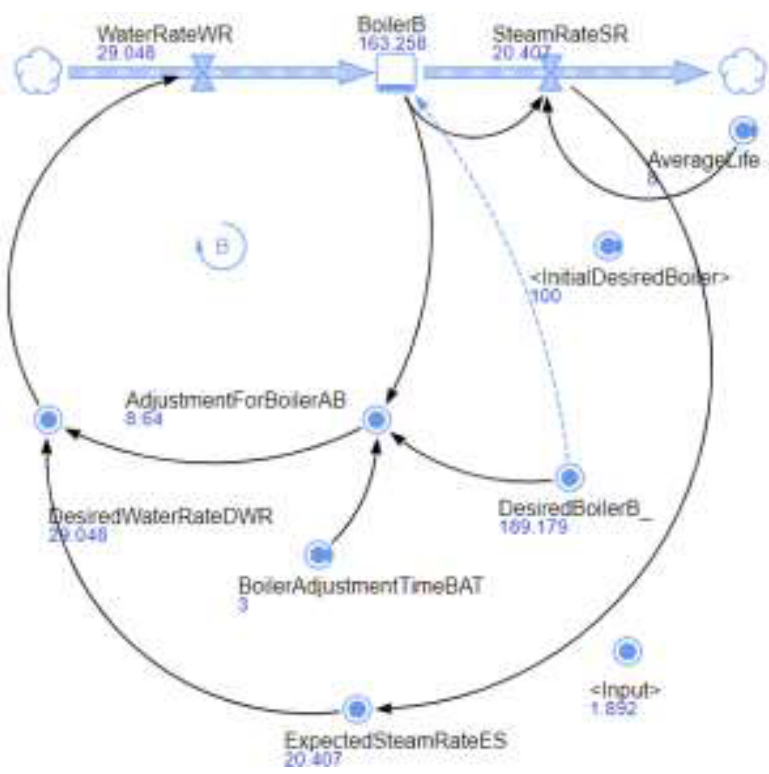

Fig. 1. A system solution for generating turbine vapor of a fuel boiler with stochastic exogenous factors.

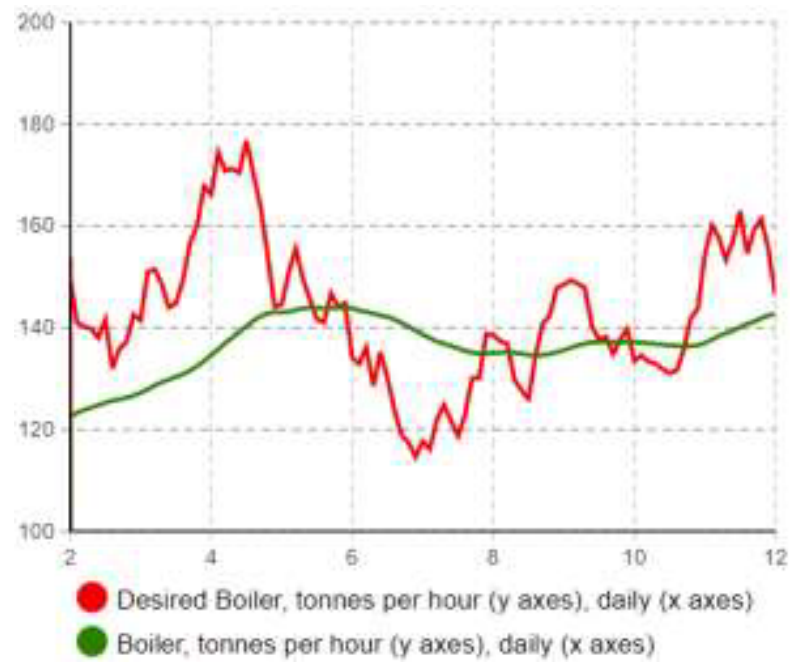

Fig. 2. The necessary and model volumes of steam generation.

Article [10] systematizes and highlights the experience of developing countries in the field of information technologies in marketing, in particular, digital marketing tools classified according to the principle of marketing needs, as well as marketing tools that are suitable and effective in a specific market context are considered.

The article [11] defines the features of "green" bonds and development practices in Europe. The number of climate-conditioned and green bonds in the world in 2018 by region, the distribution of green bond issues in Europe in 2019 by types of regional issuers and the volume of green bond issues in individual European countries in the first half of the year are also determined.

Article [12] substantiates the automation of technological process systems and control of the operation of equipment in the energy sector of the Republic of Kazakhstan, as well as the problem of automation of the entire complex of the industry, including access to networks and efficient transmission of electricity across the country. Article [13] examines a number of problems of the implementation of the state policy in the field of electric power industry, aimed at increasing energy saving So, taking into account all the pros and cons of classical types of electricity production, more and more attention is being paid to new, emerging sources - biofuels and processing of production and consumption waste. Renewable energy sources based on wind-wind energy has become the object of close attention not only of domestic entrepreneurs, but also foreign companies that consider it as a promising profitable industry for long-term investment.

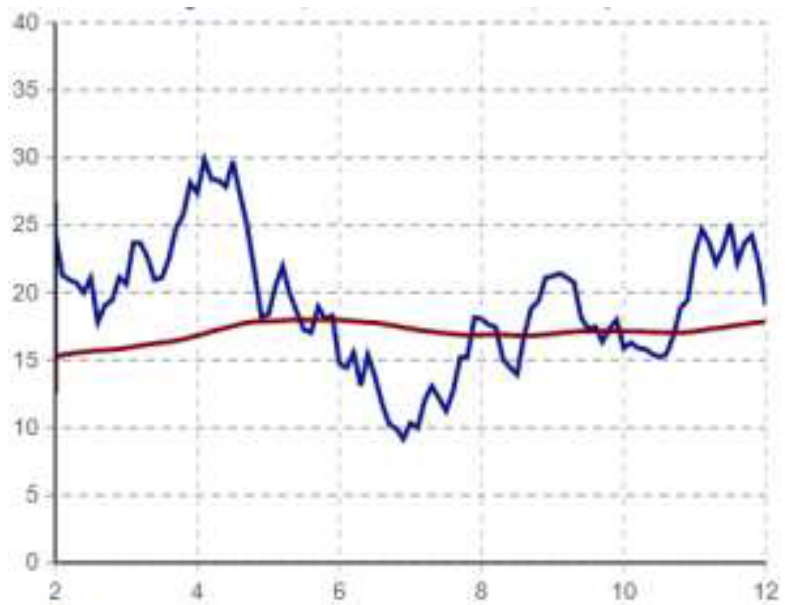

Water Rate, tonnes per hour ( $y$ axes), daily ( $x$ axes)

Steam Rate, tonnes per hour (y axes), daily ( $x$ axes)

Fig. 3. The norms of water intake and steam output of the fuel boiler.

\section{Conclusion}

Based on the analysis and review of the conceptual principles, methodology and practice of modeling water-steam processes of the fuel boiler of power plants; implementation of algorithms and models of the theory of system dynamics, white and pink noise; using computational technologies of difference-scheme equations and numerical integration methods, the values of the input parameters of the production of turbine vapor of the fuel boiler are determined), which, regardless of stochastic exogenous factors, provide stable states of the necessary and model volumes, norms of water intake and output of turbine vapor of the fuel boiler.

In conclusion, we note that the scientific results of this study can be recommended in engineering and technical solutions for the implementation of power plants using water steam in post-Soviet countries, including the Republic of Kazakhstan, according to the technical standard Stationary steam boilers: Types and basic parameters [16]. 
Acknowledgement. This research was funded by the Science Committee of the Ministry of Education and Science of the Republic of Kazakhstan (Grant No. AP09259435).

\section{References}

1. J.D. Sterman, Business Dynamics: Systems Thinking and Modeling for a Complex World. McGraw-Hill Inc. (2000)

2. A. Seefried, B. Müller, E. Förster, Journal of Sustainable Development of Energy, Water and Environment Systems, 8(1), (2019)

3. A. Capasso, A. Cervone, M.K. Falvo, R. International Journal of Electrical Power \& Energy Systems, 56, 332-339 (2014)

4. Y.-S. Jung, K. Lee, S.-Y. Lee, M.H. Choi, B.H. Lee, International Journal of Control Automation and Systems, 7(4), 615-629 (2009)

5. Y.-J. Yoon, M.H. Lee, International Journal of Control Automation and Systems, 8(6), 1241-1249 (2011)

6. T. Ancev, Water Economics and Policy, 1(3), 1550016 (2015)

7. V.C. Osma, J.E. Capilla, M.Á. Pérez-Martín, Hydrological Sciences Journal, 60(1), 1341-1354 (2015)

8. S.W. Fleming, C. Wong, G. Graham, Hydrological Sciences Journal, 59(6), 1154-1166 (2014)

9. D.M.D. Hendriks, M.J.M. Kuijper, R. van Ek, Hydrological Sciences Journal, 59(3-4), 562-577 (2014)

10. A.I. Leonow, M.N. Koniagina, S.V. Petrova, E. Grunt, S.Y. Kerimkhulle, V.G. Shubaeva, Espacios, 40 (38) (2019)

11. S. Niyazbekova, L. Moldashbayeva, S. Kerimkhulle, N. Dzholdoshev, T. Dzholdosheva, M. Serikova, E3S Web of Conferences, 244, 10060 (2021)

12. G. Azieva, S. Kerimkhulle, U. Turusbekova, A. Alimagambetova, S. Niyazbekova, E3S Web of Conferences, 258, 11003 (2021)

13. S. Niyazbekova, L. Moldashbayeva, S. Kerimkhulle, B. Jazykbayeva, E. Beloussova, B. Suleimenova, E3S Web of Conferences, 258, 11011 (2021)

14. S. Kerimkhulle, Z. Aitkozha, 1880, 040016 (2017)

15. The official site of The AnyLogic Company. https://www.anylogic.com (2021)

16. GOST $-3619-89$. Stationary steam boilers. Types and basic parameters. USSR State Standard (1989). (in Russian) 